\section{UJMM

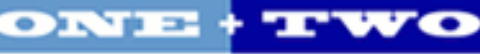

Volume 2 | 2010 Spring

\section{Undergraduate Journal of Mathematical}

Modeling: One + Two

2010

\title{
Power Consumption of a MOSFET
}

Frederick Selkey

University of South Florida

Advisors:

Arcadii Grinshpan, Mathematics and Statistics

Andrew Hoff, Electrical Engineering

Problem Suggested By: Andrew Hoff

Follow this and additional works at: https://digitalcommons.usf.edu/ujmm

Part of the Mathematics Commons

UJMM is an open access journal, free to authors and readers, and relies on your support:

Donate Now

\section{Recommended Citation}

Selkey, Frederick (2010) "Power Consumption of a MOSFET," Undergraduate Journal of Mathematical Modeling: One + Two: Vol. 2: Iss. 2, Article 11.

DOI: http://dx.doi.org/10.5038/2326-3652.2.2.11

Available at: https://digitalcommons.usf.edu/ujmm/vol2/iss2/11 


\title{
Power Consumption of a MOSFET
}

\begin{abstract}
A MOSFET is defined as metal oxide semiconductor field effect transistor. These electrical components are combined or integrated to form control and logic functions for laptop and desktop computers, power controls in printing devices, motor controls and are used in many other electrical circuits. All electrical devices consume electrical power based on current and voltage. For this paper we calculated the power consumption of a Toshiba 2SK3563 MOSFET during its triode mode by finding the area under the currentvoltage characteristic curve.
\end{abstract}

Keywords

Semiconductors, Power Consumption, Triode Mode

Creative Commons License

(c) (i)(9)

This work is licensed under a Creative Commons Attribution-Noncommercial-Share Alike 4.0 License. 


\section{TABle OF CONTENTS}

Problem Statement

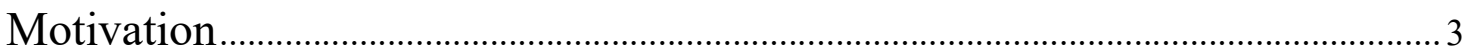

Mathematical Description and Solution Approach .....................................................

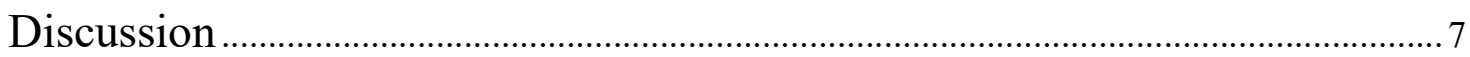

Conclusion and Recommendations..............................................................................

Nomenclature

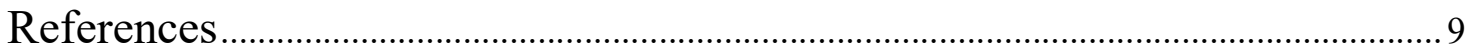




\section{PROBLEM STATEMENT}

Use the $I V$ characteristic to explore the power consumption properties of a MOSFET.

\section{MOTIVATION}

Electrical power is a vital component of society. Although energy cannot be destroyed, it does change forms and can be lost through work, friction, or dissipated as heat. As a result, there is a limited amount of energy in the form of electricity. Therefore, it is important to know how much electrical power a device consumes. Knowing where power is consumed is necessary improve efficiency and conserve valuable electricity. MOSFETs are extremely popular in electrical components.

Specifically, a MOSFET is a Metal Oxide Semiconductor Field Effect Transistor. MOSFETS are transistors which contain four terminals, namely the gate, body, source and drain. When a sufficient voltage is supplied from the gate to the body terminal, an electrical connection is opened between the source and the drain terminals.

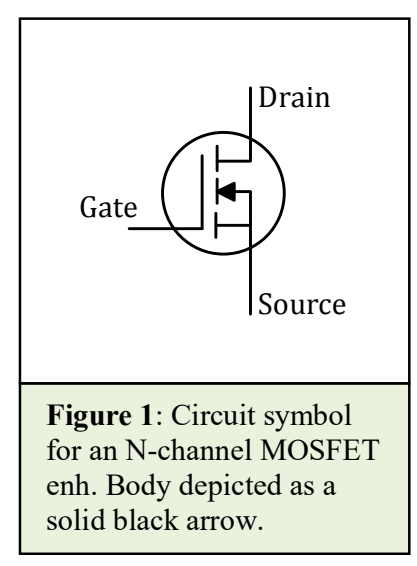

As with all electrical devices, MOSFETs consume power. The power they consume is the product of the current and voltage during operation. The physical unit for power is watts which may be expressed by any of the following forms

$$
1 \text { Watt }=(1 \text { Volt })(1 \text { Amp })=\left(\frac{1 \text { Joule }}{1 \text { Coulomb }}\right)\left(\frac{1 \text { Coulomb }}{1 \text { Second }}\right)=\frac{1 \text { Joule }}{1 \text { Second }}
$$


The integral of a function in the $I V$ (current-voltage) plane is the area under the curve which may be interpreted as the product of current and voltage. Therefore, by finding the integral of the function generated by a MOSFET, the power consumption can be determined.

MOSFETs operate in one of three stages: 1) Cutoff mode, 2) Triode mode, or 3) Saturation mode. During the cutoff mode, the gate source voltage is insufficient to activate the transistor and the connection between the source and drain terminals remain closed. During the triode mode the gate voltage is enough to establish a weak connection between the source terminal and the drain terminal. In this mode the transistor acts like a resistor whose resistance is a function of the voltage applied to the gate terminal. Finally, when sufficient voltage is applied to the gate terminal the transistor enters saturation mode and a full connection is established between the source and drain terminals.

A MOSFET in cutoff mode will consume no power as there is no connection between the source and drain terminals and a MOSFET in saturation mode consumes a fixed amount of power as the connection between the source and drain is fully open. For the remainder of this paper we explored the variable power consumption of a MOSFET during its triode mode. 


\section{MATHEMATICAL DESCRIPTION AND SOLUTION APPROACH}

The equation for the linear region of an enhancement-mode n-channel MOSFET during its triode mode is given by the equation (MOSFET - Wikipedia, the free encyclopedia, 2011):

$$
I_{D}=\mu_{n} C_{o x}\left(\frac{w}{l}\right)\left[\left(V_{G S}-V_{T H}\right) V_{D S}-\frac{1}{2} V_{D S}^{2}\right]
$$

where $I_{D}$ is the drain current, $\mu_{n}$ is the charge-carrier effective mobility, $C_{o x}$ is the gate oxide capacitance per unit area, $w$ is the gate width, $l$ is the gate length and $V_{G S}, V_{T H}$ and $V_{D S}$ are the voltages for the gate-source, threshold, and drain-source. The threshold voltage determines when a transistor changes from the cutoff to the triode mode, i.e. the transistor is in cutoff mode when $V_{G S}<V_{T H}$. For convenience, let

$$
k=\mu_{n} C_{o x}\left(\frac{w}{l}\right)
$$

Note that $k$ is based on the maximum power dissipation and gate voltage for each MOSFET.

Given a fixed gate voltage and threshold voltage, the power dissipated by a MOSFET when it is turned on is given by the area under equation (2). We considered this integral and evaluated it over the region where the drain-source voltage was considered off to a drain-source voltage where the device is considered on. Thus,

$$
\begin{aligned}
W=\int_{V_{o f f}}^{V_{o n}} I_{D} d V_{D S} & =k\left[\left(V_{G S}-V_{T H}\right) \int_{V_{o f f}}^{V_{o n}} V_{D S} d V_{D S}-\frac{1}{2} \int_{V_{o f f}}^{V_{o n}} V_{D S}{ }^{2} d V_{D S}\right] \\
& =k\left(\left(V_{G S}-V_{T H}\right)\left[\frac{1}{2} V_{D S}{ }^{2}\right]_{V_{o f f}}^{V_{o n}}-\frac{1}{2}\left[\frac{1}{3} V_{D S}\right]_{V_{o f f}}^{V_{o n}}\right) \\
& =\frac{1}{2} k\left(\left(V_{G S}-V_{T H}\right)\left({V_{o n}}^{2}-V_{o f f}{ }^{2}\right)-\frac{1}{3}\left(V_{o n}{ }^{3}-V_{o f f}{ }^{3}\right)\right) .
\end{aligned}
$$


Typically, all of the values in (4), except $k$, are supplied by the MOSFET manufacturer in a product specification sheet. For the particular MOSFET 2SK3563 manufactured by Toshiba (Toshiba 2SK3563, 2003), we have

$$
W=35 W, V_{G S}-V_{T H}=30 \mathrm{~V}, V_{\text {on }}=500 \mathrm{~V}, V_{\text {off }}=0 \mathrm{~V} \text {. }
$$

and (4) becomes

$$
35=\frac{1}{2} k\left((30)\left(500^{2}-0^{2}\right)-\frac{1}{3}\left(500^{3}-0^{3}\right)\right)
$$

which implies that

$$
k \approx-2.049 \times 10^{-6} \mathrm{~A} / V^{2}
$$

The manufacture's ratings do not contain (7). Knowing the value of $k$ makes made impossible to accurately determine the true power dissipation of this transistor during the triode mode.

Similarly, one can determine the value $k$ for any similar enhancement-mode n-channel MOSFET given its drain-source voltage, gate-source voltage, threshold voltage and power dissipation. The maximum ratings of these values are typically listed by the manufacturer. Once $k$ has been determined, the power dissipation of the device can be found explicitly for any applied drainsource and gate voltages. 


\section{DISCUSSION}

This solution provided the tools to evaluate the power consumption of an enhancementmode n-channel MOSFET with only the information provided by the manufacturer's data sheet. The value of $k$ found in (7) is the correct order of magnitude based on the physical properties of semiconductors. The method outlined in the above section can be used to find the power consumption of any similar device that has a current-voltage dependence. Furthermore, relations like (2) can be used to find values omitted by the semiconductor manufacturers. Based on the growing popularity of MOSFETs and the limited availability of electricity, it is important to know where power is consumed in an electrical device. MOSFETS popularity is due to, in part, the small amount of power required to control the device. Gate leakage is lower in MOSFETs than in other transistors such as bipolar junction transistors (BJTs).

\section{CONCLUSION AND RECOMMENDATIONS}

Determining the unpublished constant $k$ in (7) for the Toshiba 2SK3563 MOSFET allowed us to explore the electrical consumption of the component during its triode mode. The constant was estimated to the magnitude of $10^{-6} A / V^{2}$. Though the cutoff, triode, and saturation modes of modern MOSFETs are modeled using far more complicated models than that of (2), the principles we explored here can still be applied to the increasing complex models. If the power consumption of a MOSFET is determined, the total power consumption of integrated circuits can be found. 


\section{NOMENCLATURE}

PHYSICAL UNITS

\begin{tabular}{|cllc|}
\hline Symbol & Physical Property & Units & Symbol \\
\hline$P$ & Power & Watts & $W$ \\
\hline$V$ & Voltage & Volts & $V$ \\
\hline$I$ & Current & Amps & $A$ \\
\hline$E$ & Energy & Joules & $J$ \\
\hline$C$ & Charge & Coulombs & $C$ \\
\hline$T$ & Time & Seconds & $S$ \\
\hline$L$ & Length & Meters & $m$ \\
\hline
\end{tabular}

\section{LIST OF SYMBOLS}

\begin{tabular}{|clc|}
\hline Symbol & Description & Units \\
\hline$I_{D}$ & Drain-source current & $A$ \\
\hline$C_{O x}$ & Gate oxide capacitance per area & $A \cdot s /\left(V \cdot \mathrm{cm}^{2}\right)$ \\
\hline$V_{D S}$ & Drain-source voltage & $V$ \\
\hline$V_{G S}$ & Gate-source voltage & $V$ \\
\hline$V_{T H}$ & Threshold voltage & $V$ \\
\hline$\mu_{n}$ & Charge-carrier effective mobility & $\mathrm{cm}^{2} /(V \cdot s)$ \\
\hline$w$ & Gate width & $\mathrm{cm}$ \\
\hline$l$ & Gate length & $\mathrm{cm}$ \\
\hline$k$ & $\mu_{n} C_{o x}\left(\frac{w}{l}\right)$ & $\mathrm{A} / V^{2}$ \\
\hline
\end{tabular}




\section{REFERENCES}

MOSFET - Wikipedia, the free encyclopedia. (2011). Retrieved from Wikipedia:

http://en.wikipedia.org/wiki/MOSFET

Toshiba 2SK3563. (2003, January 3). Retrieved 2010, from Audio Lab of Ga:

http://www.audiolabga.com/pdf/2SK3563.pdf 